\title{
Educación Inclusiva para la Ciudadanía Europea: el doble itinerario
}

\author{
Fran J. García-García, Manuel Lopez-Torrijo, Vicent Gozálvez \\ Universidad de Valencia - España
}

\section{RESUMEN}

La educación para la participación ciudadana es fundamental para Europa en este momento. La propia Comisión Europea y el Consejo lo reconocen en varios informes acerca de la hoja de ruta en materia educativa. Sin embargo, existen múltiples barreras de aprendizaje y de participación en los sistemas educativos de los estados miembros, lo que se dibuja un doble itinerario. Todos los ciudadanos europeos necesitan unos mínimos educativos que les permitan formar parte de proyectos comunes, pero esto implica una educación para todos, en equidad, con igualdad de oportunidades para desarrollarse y aportar a la sociedad las capacidades particulares que cada persona tiene. En otras palabras: esto requiere aceptar que la educación, o es inclusiva, o no es educación. A lo largo del artículo se revisa la literatura sobre este tema, realizando una interpretación crítica con el fin de argumentar cómo la Educación Inclusiva para la ciudadanía europea beneficia a todas las personas. De hecho, la segregación o dejar de tomar en consideración a determinados ciudadanos con ciertas limitaciones nos perjudica a todos y no conviene a nadie. El trabajo concluye con propuestas de mejora para implementar el modelo educativo europeo vigente.

Palabras Clave: Educación inclusiva, participación ciudadana, capacidades, Europa, finalidad de la educación.

\section{Inclusive Education for European Citizenship: the double pathway}

\section{ABSTRACT}

Education for citizen participation is fundamental for Europe at this time. The European Commission itself and the Council recognize this in several reports on the educational agenda. However, there are multiple learning and participation barriers in the education systems of the member states, which draws a double pathway. All European citizens need a minimum education that allows them to be part of common projects, but this implies an education for all, in equity, with equal opportunities to develop and contribute to society with the particular skills each person has. In other words: this requires accepting that education is inclusive or it is not education.The literature on this topic is reviewed throughout thispaper, making a critical interpretation in order to argue how inclusive education for European citizenship benefits all people. In fact, the segregation or simply not taking into account certain citizens with certain limitations harms us all and does not suit anyone. This work concludes with proposals to improve the current European educational model.

Keywords: Inclusive education, citizen participation, capability, Europe, aims of education.

\section{Introducción}

La implicación ciudadana en los asuntos públicos es una consecuencia lógica de vivir en comunidades abanderadas por la libertad y la autonomía. No sorprende que la educación europea lubrique cada vez más los engranajes cívicos. Si el primer plan de educación de la Unión Europea,en 1976, sólo contemplaba la participación de adultos restringida al ámbito escolar, lo cierto es que las propuestas actuales inciden de lleno en el desarrollo de habilidades para la participación de todos los ciudadanos en un ámbito social más amplio. Aunque tal participación requiere unos mínimos educativos para formar parte de proyectos comunes, no siempre está claro el grado de acción cívica que puede desempeñar cada persona; y esta duda se extiende a cuestiones de justicia social para beneficio o perjuicio de todos.
Por ello es crucial dilucidar en qué medida hay diferentes itinerarios educativos para estimularla participación ciudadana de acuerdo con la formación que ofrecen los sistemas educativos en cada país. Con este motivo, el presente artículo recoge análisis referidos a los conceptos, a la financiación, a la situación de los programas para aprender a ejercer la ciudadanía, y al doble criterio en la educación formal y no-formal. El método de análisis consistió en la revisión de la literatura, la reflexión y la interpretación crítica.

En el artículo se da especial relevancia a las limitaciones de las personas de cara a su plena participación, y al sentido último de la educación como desarrollo de capacidades humanas para tal fin. Consideramosque la libertad es al mismo tiempo condición y fin de la educación (Jover, Gozálvez, y Prieto, 2017) y, en este sentido, la acción educativa es clave para la participación 
como manifestación de libertad cívica. Teniendo en cuenta que la Educación Inclusiva (EI) es motor para la plena participación, los organismos de referencia internacional reivindican una $E d u$ cación para Todos (UNESCO, 1990, 2015), una educación a la que todas las personas tengan acceso sin importar sus necesidades educativas. No es nuestro propósito revisar las concepciones generales sobre la educación en este momento, pero sí afirmarnos en una idea: la educación que no es inclusiva, no es educación. Si lo fuera, sería por definición para todo ser humano y, por tanto, sería inclusiva.

Nuestro análisis indica que la optimización del modelo educativo europeo pasa necesariamente por hacer efectiva la igualdad de oportunidades para la autonomía y la participación ciudadana. Esto podría activar la capacidad crítica y la reconstitución de valores comunes por parte de todos los ciudadanos. Es más, aprender a reflexionar acerca de las experiencias prácticas de uno mismo y a reunir un conjunto sólido de creencias seguidas de la acción incrementa las habilidades para generar un procesamiento personal de valores y de informacionesen una determinada comunidad (Dewey, 1910). No obstante, sin eliminar las barreras de acceso al aprendizaje y la participación y, con ellas, sin reducir las limitaciones de los ciudadanos, dichos valores e informaciones quedan desligados de la comunidad. Esto genera un cargo para todos que no parece ser ni justo ni conveniente para nadie.

\section{El fin de la Educación Inclusiva}

Las limitaciones forman parte de la condición humana. Aprendemos gracias a ellas y por ellas nos proyectamos sobre un continuo temporal de desarrollo individual y colectivo. De hecho, el sentido último de la EI nunca fue cubrir necesidades, sino descubrir capacidades en cualquier ser humano. Este razonamiento puede derivar de, al menos, dos argumentos: la cadena de fines educativos y las raíces normativas de la propia inclusión.

La cadena de fines educativos es jerárquica, puesto que algunos fines constituyen medios para lograr otros más lejanos. Aristóteles (2015) lo aclara así: “(...) los fines de las [artes] directivas son preferibles a los de cuantas se subordinan a ellas" (p. 58). De esta forma, cubrir necesidades individuales sirve para alcanzar aquello en que consiste la EI: suprimir barreras de aprendizaje y participación (Booth \& Ainscow, 2002), permitiendo una Educación para Todos. Sin dichas posibilidades de participación es difícil imaginar cómo alguien podría aportar sus capacidades a la construcción de lo común, sea esto público o privado.

En cuanto a las raíces normativas, el primer enclave que da paso a la inclusión en el sistema educativo es la idea de normalización (Nirge, 1969; Wolfensberger, 1972). Esto desencadenó una sucesión de reformas educativas en los años 70 en Europa y Norte América, hasta que en 1978 se publica el Informe Warnock. La propuesta es sustituir el viejo enfoque de las deficiencias por el de las necesidades especiales, aunque no conviene confundir el enfoque con el fin. Toda la reforma se engarza sobre el fin de normalizar las condiciones de las personas a través de su educación para participar en proyectos comunes,en igualdad de condiciones con los demás, hasta donde sea posible.

Más adelante se ha comprendido que la normalidad es un constructo, y los seres humanos, únicos e irrepetibles (Castillo Arenal, 2013). Lo normal, estructural y consustancial en el ser humano es desarrollar sus capacidades desde las limitaciones y necesidades que cada uno tenemos. Este es el fin último de toda educación, por supuesto inclusiva.

Si el fin es descubrir capacidades, debe haber un medio idóneo para ello.Eso implica un movimiento entre el punto de partida y el fin: una variable. La situación cambia en tanto que se avanza hacia el fin. Cualquier cambio conlleva dirección, porque hay un movimiento en un contexto. Esto constituye una función, que cobra sentido en un ambiente determinado. Así, la limitación es fruto de la interacción entre una persona y un ambiente, puesto que en ninguno de ellos acontece de manera eminentemente aislada. Entonces, la persona está sujeta a la educación, pero a veces no puede interactuar con el entorno que permite su desarrollo potencial. Siendo dicho entorno modificable, adaptarlo parece el medio más razonable para eliminar barreras de participación. Sin esas barreras, la persona podría estar menos limitada, acceder a situaciones de aprendizaje y tomar decisiones sobre su propia actividad.

Por otro lado, algunos organismos mundiales defienden el aprendizaje por competencias. Esta es la postura de la OCDE (1962) desde su primera conferencia en los años 60, una década tras la normalización y una antes de las reformas inclusivas. El Proyecto DeSeCo, con el "fin" de desarrollar competencias, ha tenido un fuerte influjo en organismos supranacionales y Europacontinúa modificando su agenda educativa en esta línea (Comisión Europea, 2016). Ahora bien, las competencias y las capacidades no son interexcluyentes. Las competencias son técnicas, por tanto, son medios. Aunque algunos las presenten como fines, incluso en su cualidad de fin están supeditadas a un fin "directivo", que es la capacidad humana. La cuestión no estriba en elegir competencias o capacidades, sino en saber dónde acaba cada una. Ambas son necesarias para un ajuste entre las personas y los contextos, para la participación en proyectos comunes.

Superar la limitación no pasa por transmitir moldes o valores preconcebidos. Se trata de una EI que haga a las personas libres -y responsables-para participar. De otra forma,la participación sería heterónoma, guiada por las manos ajenas de quienes asumirían la responsabilidad sobre ciertas personas y, por ende, también su liberad. Si así ocurriese, la EI no afectaría más que a unos miembros responsables de la comunidad y, evidentemente, dejaría de ser inclusiva. Es la incidencia de todos en el funcionamiento y devenir de una comunidad lo que conduce a pensar que la EI afecta precisamente a todos (Lopez-Torrijo, 2009); que es de interés general y no particular; y que invertir en ella -en capacidades- es para beneficio y mejora de todos. En este sentido, la libertad inclusiva que defendemos alude al planteamiento neoaristotélico de Sen (1999) y Nussbaum (2013) a favor de una libertad como desarrollo humano y de la comunidad, tanto la particular como la universal o cosmopolita.

\section{Inversión educativa en Europa: dos itinerarios}

El gasto público en EI es un valor común por razones de justicia social, pero también lo es por motivos de rentabilidad económica. Toda persona tiene derecho natural y legal a la educación $\mathrm{y}$, además, es más rentable la autonomía que la dependencia. Sin embargo, los países europeos invierten en cantidades distintas de elementos para lograr un sistema educativo inclusivo o para disponer de recursos compensatorios en las escuelas (Figura 1).

El último informe de la European Agency for Special Needs and Inclusive Education (EADSNE, 2016) sobre el gasto en EI ofrece datos de 18 territorios: 16 de la Unión Europea y 2 del Espacio de Schengen. El informe distingue entre financiación de elementos sistémicos y de provisiones adicionales para la compensación educativa. Llama la atención la discrepancia entre ambos conceptos de gasto en países como Malta o Letonia, aunque todas las zonas destacan por gastar más en elementos del sistema,con excepción de Croacia y Polonia. Ambos son precisos para superar limitaciones y desarrollar capacidades, pero entendemos que difícilmente se puede encarnar un marco específico para la EI sin 


\section{"Componentes de sistema $\quad$ Provisiones adicionales}

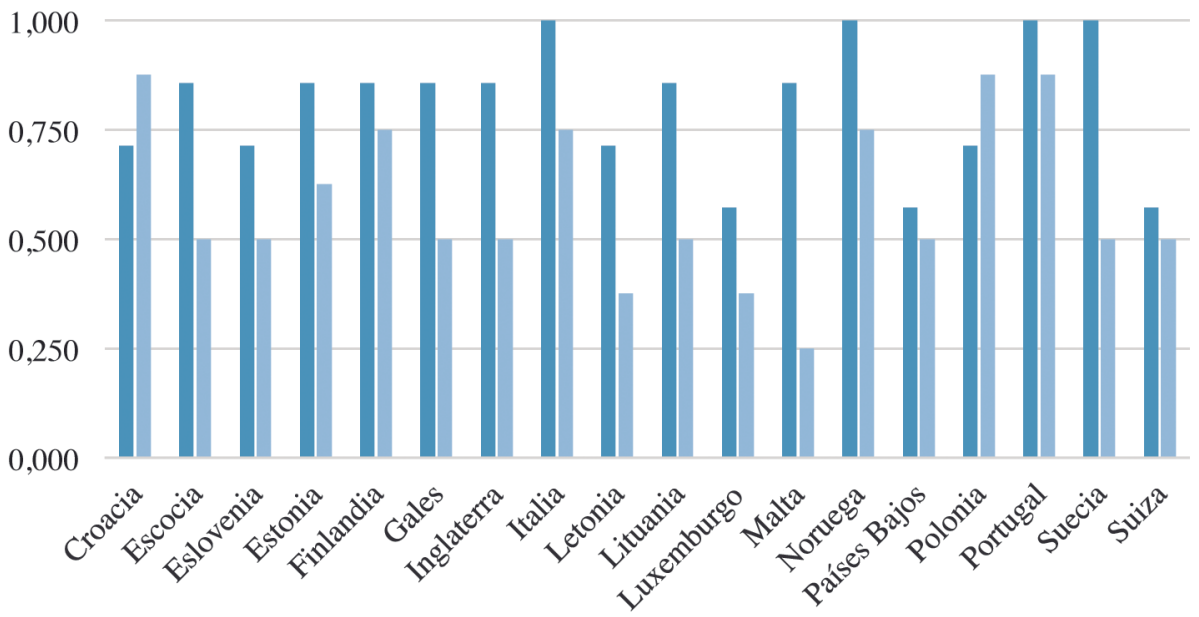

Figura 1. Frecuencia de conceptos de gasto en componentes del sistema educativo y en provisiones adicionales. Copyright 2016 por EADSNE.

contratar profesorado de asistencia, reducir ratios o disponer de ayudas técnicas.

Esto indica que no en todas partes hay iguales garantías para superar limitaciones y, por tanto, la convergencia europea no es exactamente efectiva. Se diría que existe una Europa a diferentes velocidades, donde no hay igual acceso para todos y en todo lugar. Si no hay un acceso potencial y universal al desarrollo decapacidades, la libre aportación y toma de decisiones de algunas personasen la "ciudad" está limitada. De hecho, se ha comprobado que estas barreras de participación acaban por afectar a cada miembro de una comunidad.

Se ha dicho que el ser humano tiene derecho natural y legal a la educación, y que ésta es de por sí inclusiva. Las personas están sujetas a la educación por indeterminación biológica. Parten de un estado de naturaleza ególatra -Hobbes, Locke, Rawls, etc.- con la potencialidad de adquirir una autonomía intelectual y moral, saliendo de su estado inmaduro de minoría de edad -Kant, Piaget, Kohlberg, etc.-. Ya en la antigüedad, pensadores como Hipias, Antifonte o Antidamantehabían señalado que la ciudadanía es una condición natural del ser humano, de su physis; y no se es ciudadano sólo por convención legal. Ese nomos está posteriormente atribuido y es a menudo mutable. El ser humano es educable por naturaleza y eso lo lleva a ser potencialmente cívico y a poder tomar parte en los asuntos de la "ciudad".

En esta línea, la igualdad de oportunidades para superar limitaciones reduce el riesgo de heteronomía en el sentido de la Elección Racional de Rawls (1971). No obstante, el deseo de la autonomía ajena, buscado por el bien propio, parece una aspiración todavía inmadura. Más allá deeso, se trata de ofrecer las máximas oportunidades de autonomía a todos para la convivencia ciudadana, para que todos puedan aportar y todos se nutran de unos mínimos. Las desigualdades de acceso -limitaciones- económicas, sociales, educativas o de otra clase no deben vulnerar esos mínimos por dos razones: se reducirían posibilidades de aportación general y, lo que es más importante,al no ser una situación socialmente justa, la convivencia acontecería en una organización social de riesgo que sería, sin duda alguna, indeseable para todos en algúnmomento. Frente a la vejez o una cojera provisional cualquiera se vería limitado y en situación de vulnerabilidad. En un momento así a nadie conviene una sociedad injusta y arriesgada; y con todo, es la empatía y el civismo, por encima de la conveniencia, lo que conduce a pensar en la educación para la participación ciudadana (Pérez-Pérez, 2016).
La EI para la participación ciudadana en Europa plantea, entonces, dos itinerarios. El primero es para quienes están en un momento y un lugar determinados, que les generan una limitación menor; el segundo, para quienes se ven más limitados. Para estos últimos no es un darwinismo social o la physis del más fuerte lo que excluye, sino la propia normativa de su comunidad en materia de educación para ejercer plenamente su ciudadanía. La inversión no es muy estratégica cuando se financian más cuestiones del sistema educativo y, en cambio, no se asume tanto el importe de los materiales compensatorios para efectuar cambios reales en dicho sistema. Un precio de este tránsito quebrado y sin consumar es la permanencia de barreras de participación ciudadana que sólo algunos padecen directamente y que a todos afecta de un modo u otro.

\section{Implicaciones educativas en la ciudadanía europea}

El artículo 20 del Tratado de Funcionamiento de la Unión Europea y el capítulo V de la Carta de los Derechos Fundamentales dicen que los ciudadanos europeos tienen derecho a una cierta participación. En la forma es una cuestión de derecho, pero ¿es también una cuestión de hecho, como sería deseable?

En los años 80, mientras continuaban las reformas inclusivas, se denunciaba la tecnocratización de las decisiones públicas. La aplicación de conocimientos técnicos para resolver los problemas públicos relegaba la participación ciudadana y los procesos democráticos para tomar decisiones (DeSario \& Langton, 1987). Paralelamente, los sistemas educativos se fueron cobijando en la comodidad burocrática e iban planteando el aprendizaje de competencias para la producción y distribución de bienes y servicios. Las competencias sociales y cívicas no se plantearon hasta la primera década del siglo XXI en la Unión Europea (2007), en base al aprendizaje permanente y a un modelo de ciudadano capaz de aprender con autonomía. Es una propuesta de aprendizaje técnico y procesual que se sigue monitorizando y evaluando. La dicotomía técnico-ciudadano ha llevado a la formación de unosprofesionales de alta complejidad con aplicación burocrática y, de otra parte,a la formación de ciudadanos competentes -técnicos- de complejidad elemental.

La competencia cívica que propone la Unión Europea incluye la participación ciudadana activa y el aprendizaje de "los" valores democráticos, insertando la técnica en idearios de sociedades, economías y ecosistemas sostenibles. Estos tres idearios 
pueden adoptar múltiples formatos, lo que lleva a preguntarse el fin de la participación: ciudadanía per se o ciudadanía para qué. La clave está en la posibilidad de criticar sistemas. Educar en competencias supone aprender a ejecutar técnicas procesuales y estrategias, pero no necesariamente cuestionar fines del propio sistema. La autonomía crítica toma un doble sendero dependiendo del modelo educativo: ajustarse a las necesidades del sistema económico,superando la flexibilidad que permiten las máquinas para adaptarse a los cambios (Zamora, 2017), o empoderarse para influir en el destino del sistema político, social, económico, ecológico e intercultural (Gozálvez-Pérez \& Contreras-Pulido, 2014).

Introducir al civis en la civitas conlleva una educación, además de un estatus jurídico, desde el mundo clásico y Europa no es la excepción. Pasado el tiempo de las economías postindustriales, el bienestar de los ciudadanos corre por cuenta de un Estado Relacional (Cooke \& Muir, 2012) y las responsabilidades pesan sobre quienes se asocian para dar respuesta a las demandas sociales. Un colectivo tiene unas necesidades -o limitaciones- compartidas y algunas personas afectadas por dichas limitaciones se organizan en asociaciones, generalmente sin ánimo de lucro; o unas sociedades económicas se alían para iniciar una obra social con persona jurídica de fundación. Esto genera un sistema edu- cativo no-formal, análogo a la escuela, en las mismas entrañas de una participación ciudadana focalizada en pequeñas entidades con intereses y sentidos propios. Así como la inversión en EI afecta a la educación escolar, la inversión en el Tercer Sector afecta a la educación no-formal en y para la participación ciudadana. La financiación en este caso está mediatizada por sellos de calidad, como el de la European Foundation for Quality Management, cuyos intereses de funcionamiento no tienen por qué ser iguales a los del Tercer Sector. De este modo, la búsqueda del sentido particular de la persona -capacidad- y el desarrollo del mismo -educación- no acaba de ser libre y debe adecuarse a estándares externos de excelencia y mejora continua que despersonalizan los proyectos de vida en pos de un sentido impropio. Es decir, no hay lugar para el pensamiento crítico en términos de sistema.

Al doble itinerario de la educación escolar y a la pérdida del sentido no-formal de la educación se suma la externalización de los programas educativos para la participación ciudadana. En la mayoría de países europeos hay programas extraescolares en el horario que muchos alumnos necesitan para el refuerzo escolar. Si estos programas habilitan para participar, la diferencia que generaba la inversión en EI en cuanto a igualdad de oportunidades se incrementa con programas a los que no todos pueden acceder por razones de tiempo y ya no tanto de capital (Figura 2).
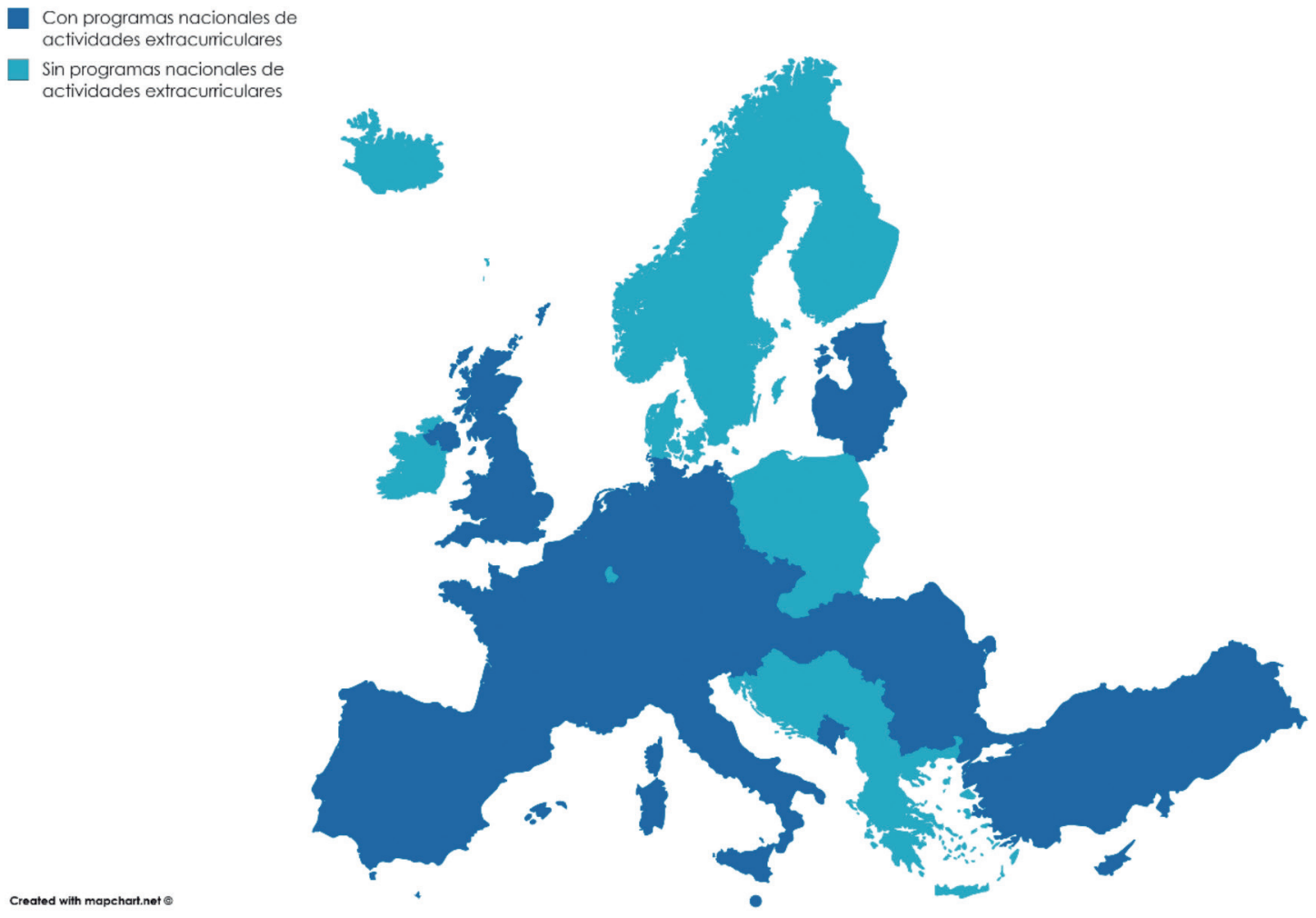

Figura 2. Programas educativos para la participación ciudadana. Copyright 2017 por Education, Audiovisual \& Culture Executive Agency (EACEA).

El Especial Eurobarómetro 437 indica que ha habido un claro aumento del número de europeos que afirma haber sufrido alguna forma de discriminación o acoso de 2012 hasta 2015 (AECEA, 2017). De hecho, el Rights, Equality and Citizenship Programme 2014-2020 tiene por primer objetivo promover la no-discriminación, que lleva reclamándose desde el siglo pasado en varias declaraciones y convenciones internacionales. El aumento de la incomodidad ciudadana y las continuas reivindicaciones no es- clarecen sino la estabilidad del doble itinerario que hunde sus raíces en el modelo educativo europeo; un modelo llamado a la mejora y a la adaptación social. En este punto, la EI es fundamental para la participación ciudadana, porque existe un requisito sine qua non para ser ciudadano: ser humano. Sólo las personas pueden ejercer la ciudadanía. Es importante ser competentes para ello, pero el desarrollo humano es absolutamente imprescindible. 


\section{Educación superior y empleabilidad}

En las sociedades europeas es necesario trabajar para poder vivir de un modo digno. Sin capital disponible es bastante más difícil conseguir comida, techo y otros recursos de primer orden, y sin embargo la vida es un derecho humano reconocido por las Naciones Unidas. Es evidente que la inserción laboral condiciona el crecimiento económico, pero también la vida de los ciudadanos, cuya capacidad genera e influye en el desarrollo de la economía. Si algún ciudadano no puede aportar sus capacidades de forma proporcionada, vagamente contribuirá con plenitud al progreso económico.

Ciertamente, este argumento es útil para justificar el aumento de bolsas de capital humano con mano de obra bien cualificada y rentable. Quizás en una Europa de mercado la participación de los ciudadanos esté supeditada a los intereses de organizaciones poderosas. El caso es queno sólo vivimos en una Europa de mercado y este argumento sirve asimismo para defender la calidad de vida y la igualdad de oportunidades. En comunidades económicamente prósperas -como son las europeas- la calidad de vida supera con creces lo que hubieran imaginado la ciudadanía de hace dos o tres generaciones. Con mayor riqueza, la escasez de recursos disminuye y la abundancia puede tener un efecto positivo en las políticas de equidad. No entraremos a valorar los efectos internacionales del mercado mundial, ni las prácticas de ciertas empresas y multinacionales en países del Tercer Mundo. Ya se sabe que la ciudadanía está más allá de las fronteras y que es cosmopolita. Pero nuestra intención es sencillamente analizar la educación para los ciudadanos europeos. En ese sentido, el doble itinerario de la educación europea conlleva un doble efecto en la inserción laboral, necesaria para ejercer efectivamente el derecho a la vida. Al final, es posible vivir sin empleo, pero la dependencia que eso conlleva en la adultez conduce lógicamente a la heteronomía. En ese punto, los criterios y normas para vivir la propia vida obedecen a la voluntad de terceros y la vida en sí deja de ser exactamente propia. Si esa es la voluntad del que vive esa vida, no hay problema. Si no lo es, el problema es que la educación no asegura la autonomía de las personas y debería ser reformulada. Tal vez un primer paso en esa dirección sea aplicar herramientas para medir la inclusión en los centros y disponemos de revisiones sobre evaluación bastante sugerentes y actualizadas (Azorín Abellán, Arnaiz Sánchez, \& Maquilón Sánchez, 2017).

Todo ser vivo se adapta al cambio para sobrevivir. De lo contrario, deja de estar vivo. El cambio en el contexto europeo es cada vez más rápido y persistente. De hecho, hay quienes lo califican como un imperativo en las sociedades europeas de hoy (Säfström, 2018). Algunos autores más influyentes hablan de sociedades líquidas (Bauman, 2000) o capitalismo flexible (Sennett, 2007). Podría decirse que vivimos en sociedades poco estables, en las que es preciso poner en práctica habilidades adaptativas. La Unión Europea (2018) ha dado una respuesta desde el aprendizaje de competencias clave y ha propuesto, en concreto, dos competencias en esta línea: aprender a aprender y adquirir un espíritu emprendedor. En realidad, no importa tanto si las competencias están más o menos abocadas al mundo de la empresa, ni tampoco si esto favorece o perjudica al conjunto de los ciudadanos. Las oportunidades para adquirir estos aprendizajes serán distintas en función del doble itinerario de la educación y eso afectará de forma notable a los valores de justicia, libertad y equidad en las sociedades democráticas de Europa. Todo lo demás no puede sino transitar hacia la desigualdad y el desequilibrio sociales, y a esto se suman los esfuerzos que se están focalizando en la educación superior para incrementar la empleabilidad.
Los Ministros Europeos de Educación Superior han ido planteando varias mejoras para lograr una mayor empleabilidad de egresados en todo el continente. En 2009 se reunieron en Lovaina y decidieron incrementar la apertura internacional del Espacio Europeo de Educación Superior para la década 20102020. Propusieron que, al menos, el 20\% de estudiantes debía haber estado un tiempo estudiando fuera de su país. Incluso se creó la Network of Experts on Student Support in Europe para ofrecer asistencia y facilitar la portabilidad de becas y préstamos para la movilidad. Esta estrategia debería provocar un aumento de las tasas de empleo tras el periodo de estudios, si no en el país propio, en otro país vecino. La cuestión es que a principios de siglo los Ministros ya se habían reunido en Praga y valoraron positivamente los planes de estudios que integraban calidad académica con empleo a largo plazo. En 2003 se volvieron a reunir en Berlín y querían garantizar las estancias en centros de otros estados con este motivo. Por tanto, es fácil pensar que la insistencia sobre este tema en la última Conferencia de París en 2018 pone de manifiesto que el objetivo de la empleabilidad no ha sido alcanzado satisfactoriamente. Eso no significa que las tasas de empleo no hayan subido, simplemente que todavía hay zonas donde tienen que subir más.

Volviendo a la inequidad de los sistemas de educación básica, sabemos que es difícil garantizar la igualdad de oportunidades para llegar a la educación superior. Suponiendo que los alumnos más desfavorecidos hayan alcanzado el nivel superior, la movilidad es un factor que aumenta la dificultad de los estudios por razones de idioma, adaptación al espacio, regulaciones, etcétera. De acuerdo con la lógica de méritos y excelencia, la movilidad se premia a la hora de conceder becas para estudios universitarios de tercer ciclo, ya en el máximo nivel educativo. Entonces, se financia la movilidad y otros presupuestos reaccionan en cadena desde ahí. Por otro lado, se reservan vacantes para estudiar y recibir ayudas a personas en situación de vulnerabilidad, como las que tienen igual o más del 33\% de discapacidad, o las que provienen de familias numerosas o monoparentales. En cualquier caso, los esfuerzos políticos y financieros para emplear a los ciudadanos están más concentrados en la educación superior, y es lógico porque se trata de las enseñanzas más técnicas y están especializadas en ciertos sectores de empleo. Pero hay muchas personas que dejan sus estudios tras la educación básica porque no pueden continuar, aunque quieran hacerlo.Esto acaba por condicionar su empleabilidad, la precariedad del tipo de empleo que encuentren -si lo encuentran- y en última instancia su calidad de vida, así como su aportación a la ciudadanía.

\section{La ciudadanía europea: conocimiento o ejercicio}

Una mirada a la plataforma de Iniciativa Ciudadana Europea ${ }^{1}$ clarifica el necesario aprendizaje de procedimientos para poner en marcha una idea: competencias digitales, autorregulación en seguimiento de instrucciones, registro online, comprensión de normas y de vocabulario de baja frecuencia léxica, etc. A ello se suma que, para ejercer los derechos como ciudadanos, hay que conocerlos previamente. Conocer y comprender estos derechos de manera universal en toda la ciudadanía europea supone quebrar el doble itinerario educativo. Eso conlleva una adecuada inversión en componentes del sistema y en provisiones adicionales, así como clarificar el fin de la educación europea, más allá el oportuno aprendizaje de competencias.

Si el fin directivo de la educación europea es el desarrollo humano-y con éste el ciudadano-, para alcanzar ese fin sería conveniente supeditar el resto de fines no-directivos a este último, que a todos los demás retroalimenta por ser, en esencia, fines hu- 
manos todos ellos. El crecimiento económico, de hecho, no existe sin componente humano que lo inicie y estabilice en tiempo y forma.Lo mismo sucede con la sostenibilidad, los sistemas políticos o las múltiples culturas europeas. Todo ello va encaminado a formar parte de proyectos comunes a través de la aportación universal de esfuerzos para su realización. Lo contrario sería extraño a los valores comunes europeos y al propio Artículo 2 del Tratado de la Unión Europea sobre inclusión y respeto a la diversidad. Por lo demás, clarificar y precisar la ruta educativa de la Unión sería conveniente más allá de conocer y ejercer los derechos de ciudadanía. De este modo, los ciudadanos podrían emprender una trayectoria hacia el futuro, que reclama un determinado tipo y medida de su participación en lo común.

Hay una relación obvia entre libertad y posibilidad que justifica la igualdad de oportunidades para la consecución efectiva de las libertades reconocidas a los ciudadanos. Evidentemente, las limitaciones sustraen libertades en diferentes rangos a unas $\mathrm{u}$ otras personas en función del momento y del lugar en donde están. Es complicado pensar cómo una educación que no permite la misma libertad potencial a todo ciudadano puede ir en contra de la discriminación y a favor de la libre participación y aportación. Por otro lado, sería legítimo que los países de la Unión Europea defendieran su soberanía, autonomía e incluso la privacidad de su accountability en el escenario de la interconexión global que transforma la naturaleza y rol del Estado. Esto llevaría a justificar las diferenciasen la inversión para una EI y, aun así, la libertad del ciudadano para participar en su comunidad es un requisito indispensable en las sociedades democráticas de hoy, situándose por encima de los derechos soberanos de cualquier gobierno representativo.

Dicha representación universal en las decisiones nacionales y supranacionales implica una mayor importancia de proteger la participación de todos y menos fuerza en decisiones representativas que se alejen de esto. La libertad está en la base de las relaciones humanas en la sociedad civil, así como lo está en las relaciones comerciales. Esto comporta intereses individuales y generales (Giddens, 1999) que condicionan la libre participación.En este punto, la libertad como ausencia de dominación (Pettit, 1997) conlleva evitar la interferencia de agentes poderosos, en cuyo caso cabría preguntarse si unos mínimos en inversión y en resultados educativos no deberían ser impuestos ni siquiera por estos organismos. Eso podría entrar en contradicción con la libertad como capacidad de asociarse de cara a la autogestión (Arendt, 1998); para actuar, decidire influir en la comunidad (Sen, 1999). En cualquier caso, los valores europeos se juzgan y reconocen por una representación parlamentaria, en vez de aflorar desde la deliberación ciudadana para conocer las posibilidades de acción social y educativa (Dewey, 2002).

Tal vez un efecto del modelo educativo es que la justicia europea esté más centrada en procesos penales que en cuestiones sociales. Una concreción de ello es la red SOLVIT para solucionar problemas informales y extrajudiciales a las empresas o a los ciudadanos; problemas que tengan que ver con reprender la protección al menor, derechos de gratuidad, la presunción de inocencia o la violencia de género. Las redes de solución de inversiones y garantías educativas para lograr mayor justicia social y capacidad ciudadana todavía están por llegar y, sin embargo, atajarían el mal de raíz. A propósito de lo penal y del conocimiento de los derechos, el Eurobarómetro de 2015 sobre ciudadanía indica que el $42 \%$ considera estar bien informado sobre sus derechos, incrementando 6 puntos porcentuales los resultados de 2012 (Unión Europea, 2017). Pero esta clase de participación como mera información del ciudadano es escasa y, no tiene tanto que ver con el desarrollo de la ciudadanía -el desarrollo humano-, como con los medios para lograrlo. Es una participación a medio tránsito, en sintonía con un modelo educativo que confunde las competencias con las capacidades en las últimas traducciones de los documentos europeos, al menos al español (p.e. Unión Europea, 2016, 2018). Seguramente todo esto ilustra la marcha y los retos de la educación que conducirían a implementar el modelo de una Europa cambiante para trascender el conocimiento de derechos hacia su pleno ejercicio.

\section{Conclusiones}

Sin duda, la educación de la ciudadanía es un tema candente y actual. En los últimos años se han publicado monográficos (Hernández Prados, 2017) y se han celebrado reuniones focales, como el Congreso Internacional de Teoría de la Educación en 2017 en Murcia. Esto denota una preocupación de la academia para abordar los retos educativos en esta línea y mejorar las condiciones actuales.

Después de todo, las tasas de abandono educativo en Europa oscilan desde el 3,1\% en Croacia hasta más del 18\% en Rumanía, España o Malta ${ }^{2}$. Parece que el reto más urgente es mejorar los sistemas educativos básicos para cubrir las limitaciones y descubrir las capacidades de todos los estudiantes. En tanto que estas limitaciones están condicionadas por el tiempo y el espacio, conviene racionalizar correctamente las partidas públicas para financiar los recursos educativos y centralizar algunas decisiones políticas que garanticen unos resultados mínimos. Hemos visto que la inversión en los centros de enseñanza para una EI es más bien poco estratégica. No sólo sería beneficiosa la racionalización de los recursos entre zonas, sino también entre los centros de un mismo país. Mientras se sigan financiando programas sin destinar fondos a conceptos de uso específico -infraestructuras, profesores de apoyo, ampliación de horarios, etc.-, será complicado implementar los sistemas de educación básica en Europa. Eso dificultará el pleno desarrollo de las personas que habitan el continente y acabará por entorpecer la participación ciudadana hasta el punto de influir negativamente en objetivos más materiales, como el crecimiento económico. Más todavía, mantener a los ciudadanos con una aportación social menor a la posibleperjudica a toda la ciudadanía y, por supuesto, abre una herida en la dignidad humana que, más allá de no ser democrática, no conviene a nadie.

Otro tanto sucede con las tasas de desempleo, que van desde el 19,1\% en Grecia o el 15,2\% en España hasta menos de los 4 puntos en los Países Bajos, Malta, Hungría, Polonia, Alemania o la República $\mathrm{Checa}^{3}$. La movilidad es una estrategia interesante para la educación superior, pero no es tan factible en los niveles básicos de la enseñanza por varias razones (edad, relación gastos-ingresos, etc.). Por eso, otros dos retos importantes son preparar a los alumnos de las enseñanzas básicas para encontrar empleo en un contexto flexible y darles a todos las oportunidades justas y necesarias para llegar con éxito a la educación superior, si así lo desean. Aunque, tanto si lo desean como si no, los sistemas educativos deberían formar ciudadanos desde el primer momento, además de empleados y empleadores. Lo contrario obstaculiza el desarrollo de la libre participación en proyectos comunes, incluyendo a las organizaciones con y sin ánimo de lucro. Hemos visto los problemas de las entidades del Tercer Sector para conservar intereses y sentidos propios, dadas las fuentes de financiación. Ante tal escenario, por qué no aprovechar metodologías docentes de vanguardia como el Aprendizaje-Servicio con la idea de transvasar recursos desde el sistema educativo reglado a la educación no-formal. Eso ayudaría a pasar de la pura crítica adaptativa a la crítica sistémica, si bien sería 
necesario el aprendizaje de técnicas. Aprender habilidades que sirvan como medios no está reñido con los fines directivos a los que éstos se orienten. Esos fines son cuestiones éticas y los medios, técnicas. Efectivamente, aprender a aprender no tiene por qué estar sólo enfocado a la adaptación de maquinaria humana para mayor producción de bienes y servicios. Puede y debería estarlo igualmente a la crítica de sistemas, la mejora social y la adquisición de valores morales hacia un estadio postconvencional como el que describía Kohlberg (1984). Este tipo de valores no se transmiten sin más; se reconfiguran constantemente en la construcción de las comunidades; y ello, no sólo es oportuno para la participación ciudadana universal, plena y real, sino que es completamente imprescindible.

Los miembros de una comunidad comparten rasgos identificadores que los diferencian de los demás (Cortina \& Conill, 2001), pero todo ser humano es naturalmente educable para convivir y aportar con sus capacidades a cualquier proyecto común. Todas las personas somos limitadas, todos caminamos hacia la perfección y jamás la alcanzamos ni remotamente. ¿Con qué derecho íbamos a negar el desarrollo de otros, cuando cada uno nos aferramos al nuestro propio tan celosamente? De eso va nuestro paso por la vida: de avanzar, de adaptarnos y realizar un proyecto en relación con otras personas. La educación -sin duda inclusiva- para la ciudadanía europea no puede mantener un doble itinerario porque no es justo, no libera, entorpece a todos y va en contra de nuestra más íntima naturaleza humana.

\section{Referencias}

Arendt, H. (1998). The Human Condition. Chicago, IL: University of Chicago Press.

Aristóteles. (2015). Ética a Nicómaco. Madrid: Alianza Editorial.

Azorín Abellán, C. M., Arnaiz Sánchez, P., \& Maquilón Sánchez, J. J. (2017). Revisión de instrumentos sobre atención a la diversidad para una educación inclusiva de calidad. Revista Mexicana de Investigación Educativa, 22(75), 1021-1045.

Bauman, Z. (2000). Liquid modernity. Malden, MA: Blackwell.

Booth, T. \& Ainscow, M. (2002). Index for Inclusion. Developing learning and participation in schools. Bristol: Center for Studies on Inclusive Education.

Castillo Arenal, T. (2013). Déjame intentarlo. La discapacidad: Hacia una visión crítica de las limitaciones humanas. Torrelavega: Amica.

Comisión Europea. (2016). Competence frameworks: the European approach to teach and learn 21st century skills. Recuperado de: https://bit.ly/2P9ekb2

Cooke, G. \& Muir, R. (Eds.) (2012). The relational state. How recognising the importance of human relationships could revolutionise the role of the state. London: Institute for Public Policy Research.

Cortina, A. \& Conill, J. (2001). Educar en la ciudadanía. Valencia: Instituto Alfonso el Magnánimo.

DeSario, J. \& Langton, S. (Eds.) (1987). Citizen participation in public decision making. New York: Greenwood Press.

Dewey, J. (1910). How we think. Boston: Heat.

Dewey, J. (2002). Human Nature and Conduct. North Chelmsford, MA: Courier Corporation.

EACEA. (2017). Citizenship Education at School in Europe - 2017. Eurydice Report. Luxembourg: Publications Office of the European Union.

EADSNE. (2016). Financing of Inclusive Education: Mapping Country Systems for Inclusive Education. Odense: EADSNE.

Giddens, A. (1999). The Third Way: The Renewal of Social Democracy. Hoboken, NJ: Wiley.
Gozálvez-Pérez, V. \& Contreras-Pulido, P. (2014). Empoderar a la ciudadanía mediática desde la educomunicación. Comunicar, 21(42), 129-136. doi:10.3916/C42-2014-12

Hernández Prados, M. A. (Coord.). Educación para la vida ciudadana en una sociedad plural. Murcia: Editum.

Jover, G., Gozálvez, V., \& Prieto, M. (2017). Una filosofía de la educación del siglo XXI. Madrid: Síntesis.

Kohlberg, L. (1984). The psychology of moral development: the nature and validity of moral stages. New York, NY: Harper \& Row.

Lopez-Torrijo, M. (2009). La inclusión educativa de los alumnos con discapacidades graves y permanentes en la Unión Europea. Revista Electrónica de Investigación y Evaluación Educativa, 15(1), 1-20. https://ojs.uv.es/index.php/RELIEVE/article/ view/4183

Nirje, B. (1969). The Normalization Principle and its human management implications. In R. Kugel and Wolfensberger (Eds.). Changing patterns in residential services for the mentally retarded (pp.179-195). Washington: Presidents on Mental Retardation.

Nussbaum, M. C. (2013). Creating Capabilities. The Human Development Approach. Cambridge, MA: Harvard University Press.

OCDE. (1962). Policy Conference for Economic Growth and Investment in Education. Paris: OCDE.

Pérez-Pérez, C. (2016). Educación en valores para la ciudadanía. Estrategias y técnicas de aprendizaje. Bilbao: Desclée de Brouwer.

Pettit, P. (1997). Republicanism: A Theory of Freedom and Government. New York, NY: Oxford UniversityPress.

Rawls, J. (1971). A theory of justice. Cambridge: Belknap Press of Harvard University Press.

Säfström, C. A. (2018). Liveable life, educational theory and the imperative of constant change. European Educational Research Journal, 17(5), 621-630. doi:10.1177/1474904118784480

Sen, A. (1999). Development as freedom. New York, NY: Anchor Books.

Sennett, R. (2007). The Culture of the New Capitalism. New Haven, CT: Yale University Press.

UNESCO. (1990). Declaración Mundial sobre Educación para Todos. Recuperado de http://unesdoc.unesco.org/images/0012/001275/127583s.pdf

UNESCO. (2015). La educación para todos, 2000-2015: logros y desafíos. París: UNESCO.

Unión Europea. (1976). Resolution of the Council and of the Ministers of Education, meeting within the Council, of 9 February 1976 comprising an action programme in the field of education. Recuperado de https://bit.ly/2QtTKTG

Unión Europea. (2007). Recommendation of the European Parliament and of the Council of 18 December 2006 on key competences for lifelong learning. Recuperado de: http://eur-lex. europa.eu/eli/reco/2006/962/oj

Unión Europea. (2016). Comunicación de la Comisión al Parlamento Europeo, al Consejo, al Comité Económico y Social Europeo y al Comité de las Regiones una nueva agenda de capacidades para Europa. Trabajar juntos para reforzar el capital humano, la empleabilidad y la competitividad. Recuperado de https://bit.ly/2yo7S6x

Unión Europea. (2017). Informe sobre la ciudadanía de la UE 2017. Fortaleciendo los derechos de los ciudadanos en una Unión de cambio democrático. Luxemburgo: Oficina de Publicaciones de la Unión Europea.

Unión Europea. (2018). Commission Staff Working Document Accompanying the Proposal for a Council Recommendation on Key Competences for LifeLong Learning. Recuperado de https://bit.ly/2J3KfnO

Wolfensberger, W. (1972). The Principle of Normalization in Human service. Toronto: National Institute on Mental Retardation. 
Zamora, J. A. (2017). El "valor" de educar: una aproximación a relación entre educación y ciudadanía. En R. Mínguez Vallejos (Presidente), Libro de Ponencias en el XIV Congreso Internacional de Teoría de la Educación 2017 (pp. 15-33). Murcia: Universidad de Murcia.

http://ec.europa.eu/citizens-initiative/public/welcome?lg=es

Datos de Eurostat, 2017: https://bit.ly/2MYID4q

Datos de Eurostat, 2018: https://bit.ly/2MRmyj6 